Document downloaded from:

http://hdl.handle.net/10251/84393

This paper must be cited as:

Triana-Infante, CA.; Pastor Abellán, D.; Varón, M. (2016). Enhancing the Multiplexing Capabilities of Sensing Networks using Spectrally Encoded Fiber Bragg Grating Sensors. Journal of Lightwave Technology. 34(19):4466-4472. doi:10.1109/JLT.2016.2537362.

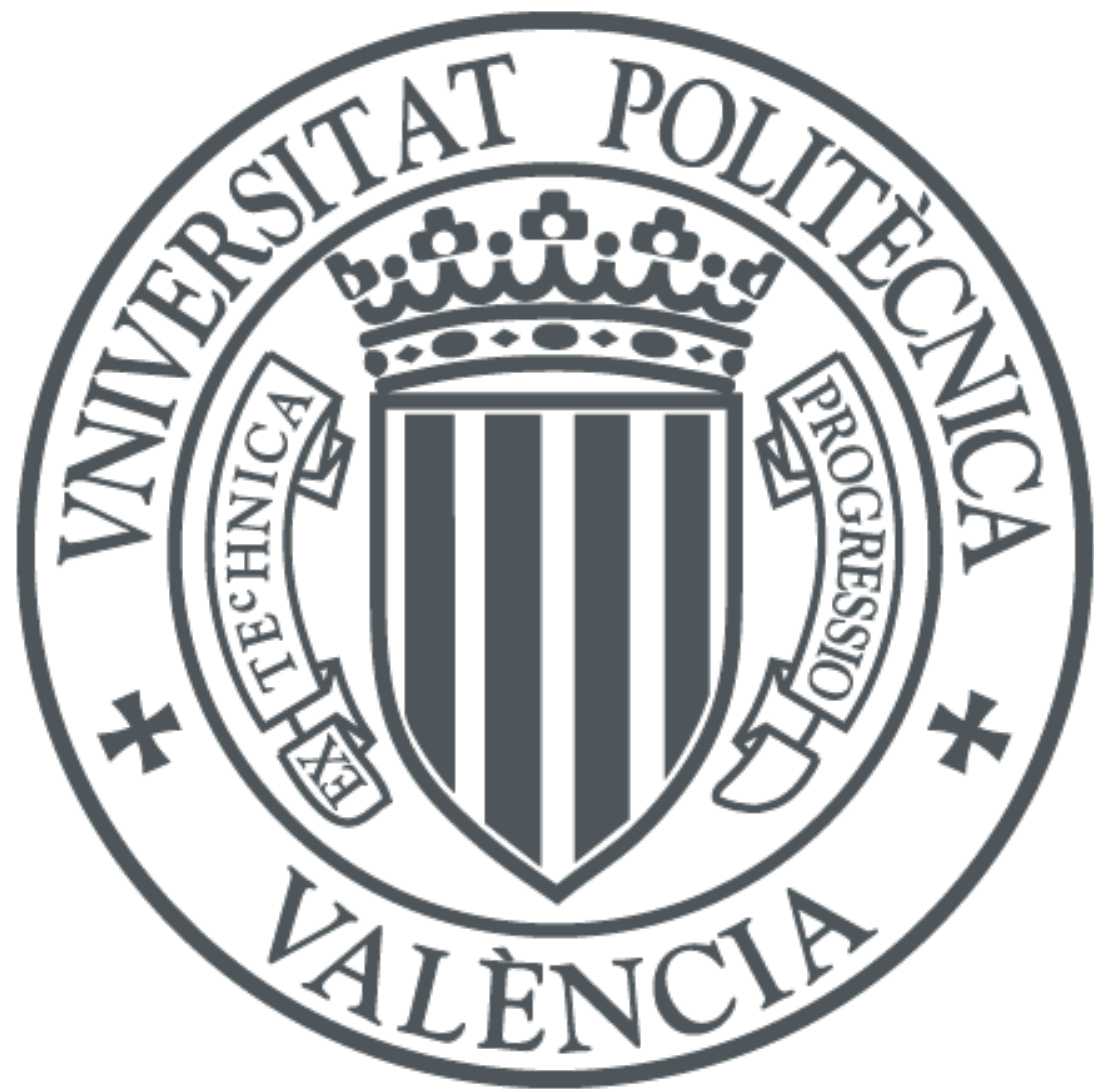

The final publication is available at

http://dx.doi.org/10.1109/JLT.2016.2537362

Copyright Institute of Electrical and Electronics Engineers (IEEE)

Additional Information

(c) 2016 IEEE. Personal use of this material is permitted. Permission from IEEE must be obtained for all other users, including reprinting/ republishing this material for advertising or promotional purposes, creating new collective works for resale or redistribution to servers or lists, or reuse of any copyrighted components of this work in other works. 


\title{
Enhancing the Multiplexing Capabilities of Sensing Networks using Spectrally Encoded Fiber Bragg Grating Sensors
}

\author{
Andrés Triana, Daniel Pastor, Member, IEEE, and Margarita Varón, Member, IEEE
}

\begin{abstract}
We have proposed a technique to design spectrally encoded FBG sensors allowing overlapping between two or more sensors in the same spectral region. A theoretical description of the design and the identification function for the proposed sensors has been developed. Simulation of the encoded FBG sensors was carried out to validate their overlap-proof behavior. Finally, the proposed sensors were manufactured and validated experimentally to show the compatibility between our encoding technique and traditional WDM multiplexing. Furthermore, the error of the proposed interrogation system was measured and discussed.
\end{abstract}

Index Terms-Fiber Bragg grating (FBG), optical fiber sensor, spectral encoding, demodulation, overlap.

\section{INTRODUCTION}

$\mathbf{O}$ PTICAL fiber sensors have been intensively studied over the past years due to their inherent characteristics such as, lightweight, immunity to electromagnetic interferences, low losses, etc. FBGs are between the most accepted sensing technologies due to their simple and linear behavior [1], [2] also, FBG sensors are naturally multiplexed in WDM since each sensor works over a fixed spectral range. Other multiplexing schemes have been demonstrated, being the TDM [3] and OFDR [4] techniques the most commonly used since they are compatible with WDM schemes, however, they increase considerably the complexity in the interrogation scheme.

As the sensing parameter of FBG sensors is the wavelength shift, the total spectrum must be divided into the operational range for each sensor in the sensing network (even considering guard-bands in between) in order to avoid overlapping between two adjacent sensors. This sets a limit to the number of sensors available in the sensing network. In order to solve the overlapping between FBG sensors, a variety of approaches have been proposed, considering optimization techniques [5] or intelligent algorithms [6], [7] for discriminating the central wavelength corresponding to each sensor during an overlapping situation. Nevertheless, this approaches are usually complex to implement in real time.

Other approaches have been carried out to involve concepts of Optical Code Division Multiple-Access (OCDMA) in a sensing system. In [8]-[11] for example, it is proposed to

Andrés Triana and Daniel Pastor are with the Optical and Quantum Communications Group (iTEAM Research Institute), Universidad Politécnica de Valencia, Valencia 46022, Spain (e-mail: catrianai@unal.edu.co)

Andrés Triana and Margarita Varón are with the CMUN Research Group, Universidad Nacional de Colombia. Cra 30 No. 45-06, 111321 Bogotá DC, Colombia

Manuscript received; modulate the source signal illuminating the sensors network with a code signal, then the detection of each sensor in the network is performed by the auto-correlation in time domain between the reflected signal and the modulating signal. We have proposed the use of an encoding technique to define the spectral shape of the sensors that allows the complete identification of two adjacent FBG sensors in the spectrum [12]. Our technique is based on optical CDMA communication systems, where a codeword is assigned to each user in the communication system to encrypt their information. So we use the well-known Optical Orthogonal Codes (OOC) to define the spectral shape of FBG sensors.

The main advantage of using spectral encoding to shape FBG sensors is that each sensor is provided with a unique signature so they can be unequivocally identified (this shape can also be associated to a spatial location in the sensing network). In this way, overlap between adjacent sensors is allowed and consequently the number of sensors in the network is increased. Also, when adding compound shapes to FBG sensors their measurement will gain robustness against interferences in the spectrum which improves the accuracy of the system.

In this paper, the feasibility of spectrally encoded FBG sensors is demonstrated, specially their compatibility with WDM multiplexing. A theoretical description on the sensors shape is provided along with the proper identification function to demodulate the wavelength of each encoded FBG sensor. Our analysis approach is completed with the experimental manufacturing and characterization of the proposed sensors. The encoded sensors were implemented by writing SuperImposed FBGs (SI-FBGs) according to a specific codeword allowing to perform experimental validation of different overlapping scenarios.

\section{PRINCIPLE OF SPECTRALLY ENCODED FBG SENSORS}

The limitation in the number of conventional FBG sensors allocated in the total source spectrum is defined by the operational range of each sensor, this is because conventional FBG sensors are not meant to overlap. Aiming at solving the overlapping limitation of FBG sensors, we have used optical orthogonal codes (OOC) in order to shape FBGs' spectral response, as a result we obtain encoded FBG sensors that can be identified even under overlapping conditions.

With the encoding of FBG sensors it is possible to identify the wavelength of two or more sensors overlapping in the 


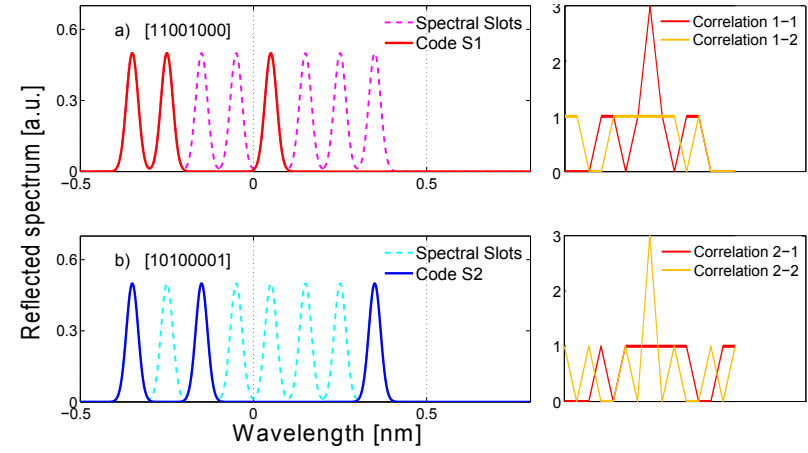

Figure 1. A set of two encoded FBG sensors are represented, according to the orthogonal codes: $S 1=$ [11001000] in a) and $S 2=$ [10100001] in b), the right plot to each sensor represents their auto- and cross-correlation function.

spectrum, i.e. more than one sensor is allowed to operate in the same spectral region, multiplying the number of sensors allocated in the same spectrum.

\section{A. Optical Orthogonal Codes (OOC)}

OOCs have been widely used in Optical Code Division Multiple Access (OCDMA) communications systems to encode temporarily the information from multiple users. In this work OOCs are used to define spectrally the shape of FBG sensors in such a way that each encoded FBG sensor is completely recognizable, using the proper decoder, from its neighboring sensors within the system. However, unlike communication systems where long orthogonal codes are required for a large number of users, we are interested in short orthogonal codes occupying a spectral portion as small as possible.

For a set of codes to be considered orthogonal, the following condition must be satisfied: each code must be completely distinguishable from shifted versions of itself and from every other code in the set. This means that the cross-correlation product, for any code with itself, must have a high autocorrelation peak (ACP) at the initial position and low side lobes at the remaining positions, and, the cross-correlation (XC) product (between two different codes) will be constrained to a low value for all the positions.

Taking into account the above considerations we selected sets of 2 and 3 OOCs through the combinatorial method described in [13], this selection was performed in order to maintain short the number of bits (also called 'chips') in the code, the higher the number of orthogonal codes in a set, the longer the codeword. The encoded FBG sensors corresponding to a set of 2 orthogonal codes are depicted in Fig. 1, in this case, the orthogonal codewords are composed of 8 chips, three of them are nonzero chips (i.e., '1'). These codewords define the spectral configuration of the proposed sensors, meaning that sensor 1 in Fig. 1 a), with code $S 1=$ [11001000], features 8 equally spaced spectral slots with reflection sub-bands assigned to each nonzero chip in the codeword. The same holds for the second sensor, $S 2=$ [10100001], represented in Fig. 1 b). The plot to the right of Fig. 1 a), b) represents the cross-correlation product between the two codewords, it is shown the ACP in the central position (where equal codewords match) and a low XC value along the spectrum for different

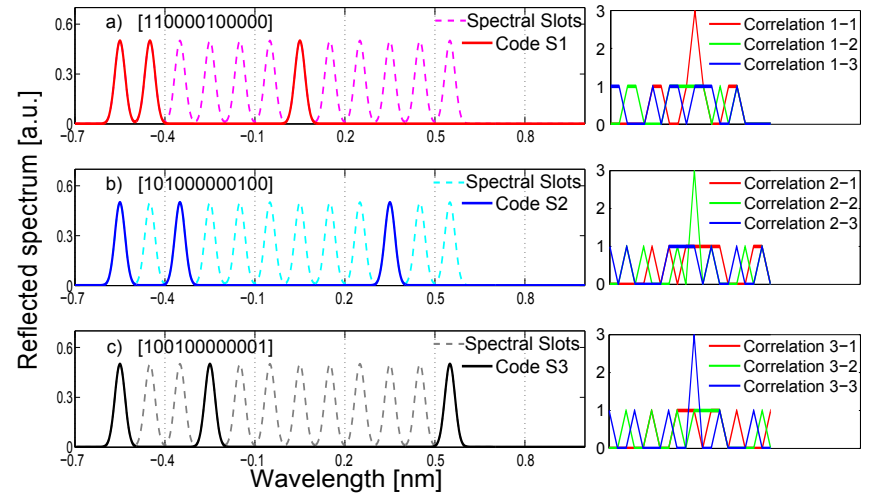

Figure 2. A set of three encoded FBG sensors is represented, according to the orthogonal codes: $S 1=$ [110000100000] in a), $S 2=$ [101000000100] in b) and $S 3=[100100000001]$ in c). The right plot to each sensor represents their auto- and cross-correlation function.

codewords. The ACP/XC ratio is constrained to one third of the total power.

In Fig. 2 it is depicted a set of 3 orthogonal sensors, in this case the codewords feature 3 reflection sub-bands allocated in 12 spectral slots. The codewords are $S 1=[110000100000]$ in Fig. 2 a), $S 2=[101000000100]$ in Fig. 2 b) and $S 3=$ [100100000001] in Fig. 2 c). Each plot to the right in Fig. 2 represents the cross-correlation product for the codewords in this set, again, The $\mathrm{ACP} / \mathrm{XC}$ ratio is constrained to one third of the total power.

The number of orthogonal sensors in a set has a direct relationship with their spectral length, however, there are other parameters affecting the spectral shape of the sensor, such as the reflectivity, $F W H M$ of the reflection sub-bands and spacing between sub-bands. Considering this, we can write down the total spectrum $R(\lambda)$ for a set of encoded sensors as (1):

$$
\begin{aligned}
& R(\lambda)= \\
& \sum_{j=1}^{K} \sum_{i=-N / 2+1}^{N / 2} c_{j}(i) \cdot R^{\prime} \cdot g\left(\lambda-\lambda_{B j}-\Delta \lambda_{B j}-(\delta \lambda \cdot i-\delta \lambda / 2)\right)
\end{aligned}
$$

Where, for a number of sensors $K$, with $N$ sub-bands. The bit-code $c_{j}(i)$ represents each reflection sub-band on a sensor, $g(\lambda)$ is the spectral shape of the sensors, with reflectivity $R^{\prime}$ and Bragg wavelength $\lambda_{B j}, \delta \lambda$ is the spectral separation between sub-bands. Increment on the $N$ sub-bands goes from $-N / 2+1$ to $N / 2$ allowing the codewords to be even or odd.

To demodulate the encoded FBG sensors it is computed the correlation product between the theoretical spectral response of the sensor and the total reflection spectrum recorded with an OSA (similar to the approach presented in [14], but in our case the spectral shape of the sensors have been previously encoded), obtaining the ACP indicating the central position $\lambda_{B j}+\Delta \lambda_{B j}$ of the corresponding sensor in the spectrum, and a constrained $\mathrm{XC}$ value for the remaining spectral positions. This demodulation can be expressed as the Identification Function of the encoded FBG sensors $(I F)(2)$. 
TABLE I

SIMULATION PARAMETERS

\begin{tabular}{lll}
\hline \hline Parameters & Symbols & Values \\
\hline Spatial Resolution & Res & $1 \mathrm{pm}$ \\
Reflectivity & $\mathrm{R}$ & 0.5 \\
Sub-bands' width & FWHM & $0.04 \mathrm{~nm}$ \\
Slot spacing & $\delta \lambda$ & $0.1 \mathrm{~nm}$ \\
Number of Sensors & NoS & 3 \\
Connection type & Config. & Parallel / Serial \\
\hline
\end{tabular}

The identification function $\operatorname{IF}(\lambda)$ is computed over the entire measured spectrum for each one of the $K$ encoded sensors. This is a simple scalar product which does not add complexity to the interrogation process.

\section{B. Simulation of encoded FBG Sensors}

As mentioned in II-A, the OOC is not the only determining factor of the spectral shape in encoded FBG sensors. In fact, parameters such as the sub-bands width, reflectivity and spacing between chips could impact both, the detection process and the manufacturing feasibility of the devices. In order to evaluate theoretically the performance of the encoded FBG sensors under overlapping conditions several simulations were performed. It is possible not only to prove the autocorrelation identification function, from equation (2), under overlapping scenarios, but also to determine the relevance of each design parameter in the wavelength detection. In Fig. 3 it is depicted the corresponding reflection spectrum for the set of 3 orthogonal sensors presented in Fig. 2. In the simulation, performed with the parameters listed in Table I, the three sensors follow linear paths that overlap over the same spectral region. Fig. 3 a) shows the total reflection spectrum and in Fig. 3 b) there are depicted the computed $\operatorname{IF}(\lambda)$ for each sensor, the ACP obtained corresponds to the central position of each encoded FBG sensor.

Fig. 4 depicts the path followed by each sensor in an overlapping sensing scenario. Detection of the central wavelength is performed for each sensor; Fig. 4 a), b) and c) represents the resulting ACP from the $I F(\lambda)$ of sensors $S 1, S 2$ and $S 3$ respectively. The insets show a zoom of the maximum error in the detection, which is of $\pm 7 \mathrm{pm}$. This detection error is caused by the $\mathrm{XC}$ values corresponding to the remaining sensors in the evaluated spectrum that are occasionally added to the expected ACP from the matching sensor.

These results confirm theoretically the overlap-proof behavior of the encoded sensors, furthermore, it was studied the effect of the shape parameters for the proposed sensors such as the sub-bands' width and the reflectivity. It was found a
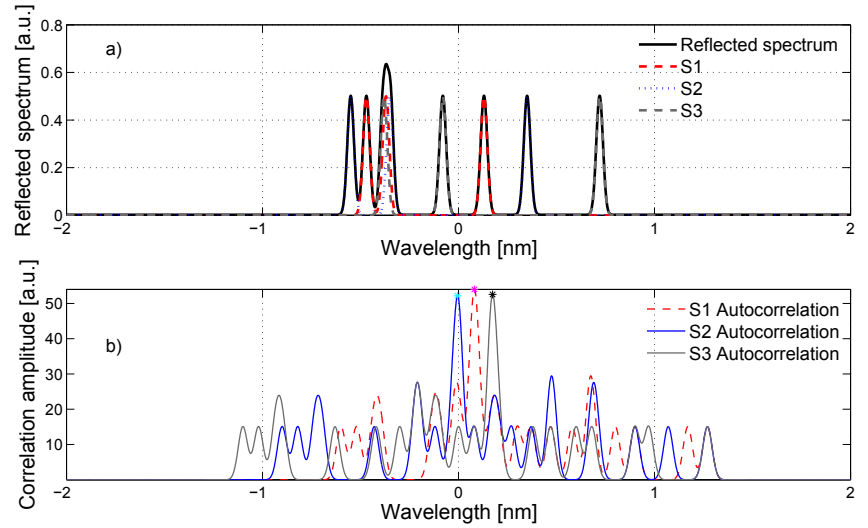

Figure 3. In a), the total reflected spectrum is simulated for the set of three orthogonal sensors. In b), their correlation product is computed in order to obtain the ACP corresponding to the central wavelength for each sensor.
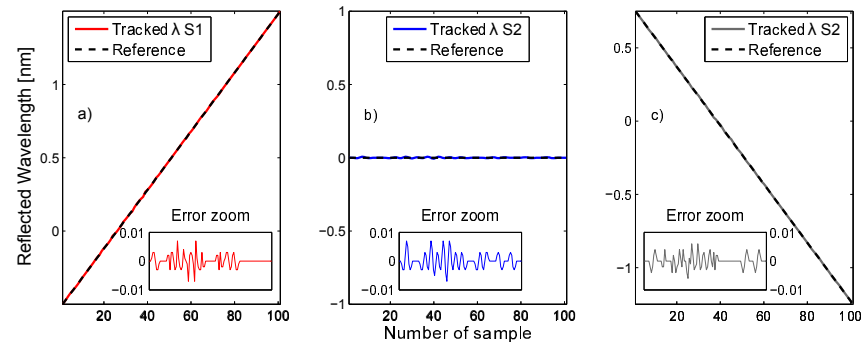

Figure 4. Trajectories followed by the three orthogonal sensors during the simulation. In a) it is plotted the trajectory for the sensor $S 1$, in b) for the sensor $S 2$ and c) shows the trajectory followed for the sensor $S 3$ during an overlapping scenario. The insets show the obtained error in the wavelength tracking.

direct relationship between the detection error and the width of the reflection sub-bands. Broader reflection sub-bands will result in a broader cross-correlation product, causing larger interference between the ACP and the XC. Regarding the reflectivity values for the encoded FBG sensors, the only limitation comes when working in serial configuration, where low-reflectivity regime is necessary to avoid adding error to the detection due to shadowing effect between sensors working at the same wavelength.

From these results, the desired features for encoded FBG sensors are: low reflectivity, in order to allow serial configuration of the sensors in a sensing network; narrow spectral width of the sub-bands to minimize the detection error caused by ACP/XC interferences, and finally, short values for the subbands' spacing $\delta \lambda$ with the purpose of reducing the spectral length of the sensors.

\section{IMPLEMENTATION AND RESULTS}

After the validation under overlapping scenarios through simulation as presented in section II-B, the encoded FBG sensors were manufactured by writing multiple gratings at the same spatial location of the optical fiber (Super Imposed FBGs, SI-FBGs [15]). Thus, resulting devices feature multiple reflection bands at the different wavelengths determined by the OOC. In order to perform a experimental validation, the set of 2 orthogonal encoded sensors (Fig. 1) was manufactured. 


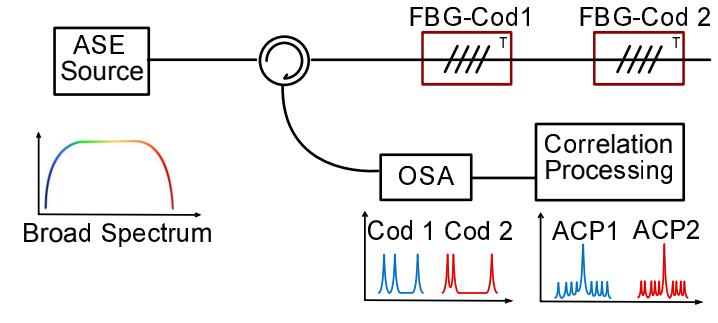

Figure 5. Serial-schematic setup used to measure the reflection spectrum of the encoded FBG sensors.

These devices were first synthetized using the Discrete Layer Peeling method (DLP) [16], obtaining a complex index profile which is executed in a point by point writing procedure by an UV laser beam reaching the photosensitive optical fiber through a Phase Mask (PM). In this way, each sensor is constrained to a spatial region in the fiber, determined by the length of the codeword, the FWHM and the distance between sub-bands.

Manufactured encoded FBG sensors are $42 \mathrm{~mm}$ long, with wavelengths $S 1=1546.5 \mathrm{~nm}$ and $S 2=1547.3 \mathrm{~nm}$ at room temperature. The reflectivity was set to $30 \%, F W H M=$ $40 \mathrm{pm}$ for each sub-band and the distance between chips $\delta \lambda=0.1 \mathrm{~nm}$

\section{A. Experimental Setup}

Experimental validation of the manufactured sensors is carried out according to the schemes presented in Figs. 5, 6, in general, a broadband source illuminates the sensing array, where each FBG device is stabilized in temperature. The total reflection spectrum is recovered through a circulator and recorded by an Optical Spectrum Analyzer (OSA) with $10 \mathrm{pm}$ resolution. Each readout is transferred to a computer in order to run the correlation identification algorithm obtaining the central position for each sensor in the network.

In the serial configuration (Fig. 5), besides low reflectivity values to avoid shadowing effect between sensors at the same wavelength, similar values of sensors' reflectivity must be guaranteed i.e., losses or low reflectivity values for one of the sensors will reduce its $\mathrm{ACP} / \mathrm{XC}$ ratio adding error to the detection process. A simple experiment of wavelength detection for a set of two orthogonal encoded FBG sensors in serial configuration is presented in Fig. 6, where one sensor is at a fixed spectral position while the second one is stretched and swept over the spectrum.

The same experiment is performed for parallel configuration of the sensors in the sensing array (Fig. 7). In this case it was used a passive 50:50 splitter, therefore the reflectivity of the sensors can be maximized in order to improve the signal to noise ratio (SNR). Results of the experiment are shown in Fig. 8 which prove the distinction capabilities of the system in an overlapping scenario.

These experiments prove the sensors' orthogonality and the distinction capability of the system. In this situation, the number of sensors in an optical sensing network is increased because every pair of sensors allows wavelength detection over the same operational range. In fact, taking full advantage of
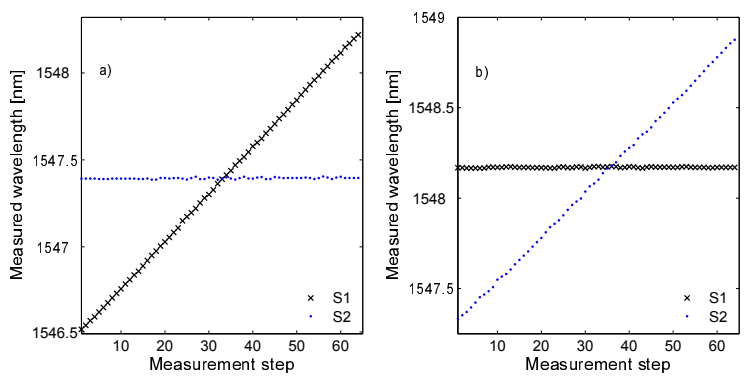

Figure 6. Measured wavelength for two sensors under overlapping conditions in serial configuration. a) shows the swept of sensor $S 1$ over the sensor $S 2$ and b) shows the analog scenario. Complete identification of the sensors' wavelength is achieved.

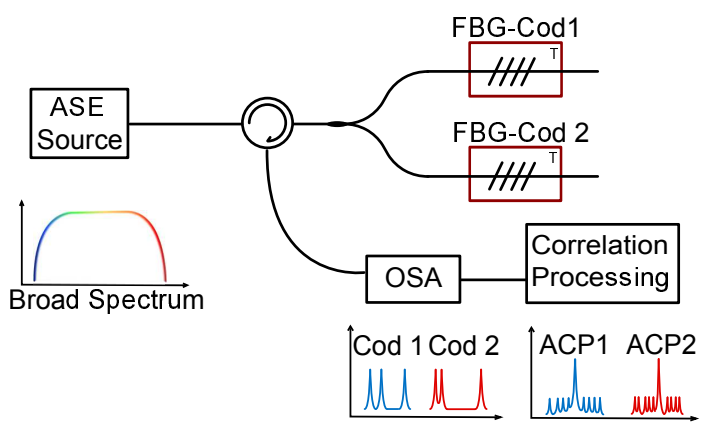

Figure 7. Parallel-schematic setup used to measure the reflection spectrum of the encoded FBG sensors.

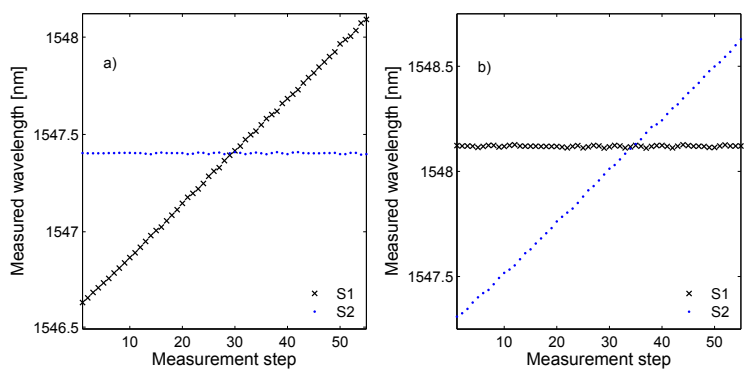

Figure 8. Measured wavelength for two sensors under overlapping conditions in parallel configuration. a) shows the swept of sensor $S 1$ over the sensor $S 2$ and b) shows the analog scenario. Complete identification of the sensors' wavelength is achieved.

the orthogonal properties of the proposed sensors (a codeword is distinguishable from other codewords in the same set and from shifted versions of itself), it is possible to deploy more than once an encoded FBG sensor in the same operational range. For example, in Fig. 9 it is shown the total reflection spectrum from 3 encoded sensors, (two sensors $S 1$ and one sensor $S 2$ ), and its resultant correlation product, where the obtained ACP points out the central wavelength of each sensor while their reflection spectra are merged. In Fig. 10 it is plotted the trajectory of each sensor and their overlapping regions, operational range of sensor $S 2$ gets overlapped at both sides by the operational range of a $S 1$ sensor, this is possible because sensors with the same code can overlap partially and still the detection procedure do not lose their reference.

This demonstrates the flexibility of the encoded FBG sensors and the compatibility between CDM and WDM, another 

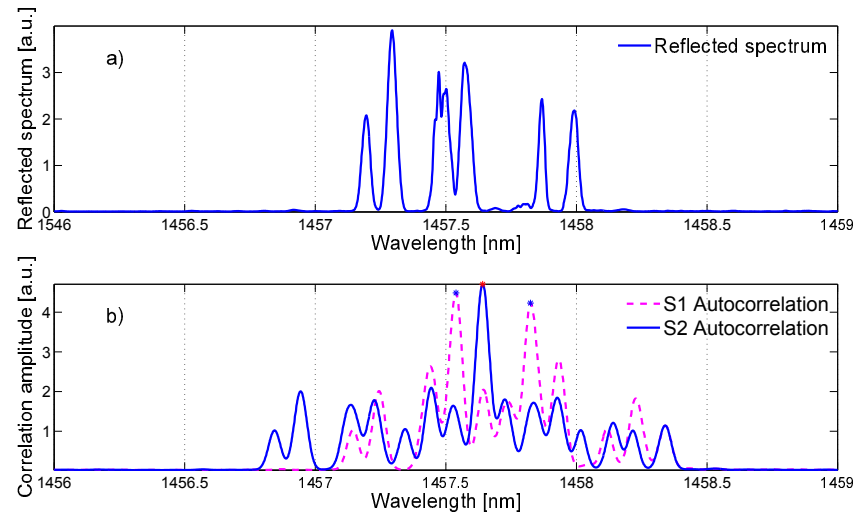

Figure 9. Experimental measurement of the reflection spectrum from three encoded FBG sensors. In a), a readout of the total reflected spectrum under overlapping conditions is measured. In b), their correlation product is computed in order to obtain the ACP corresponding to the central wavelength for each sensor.

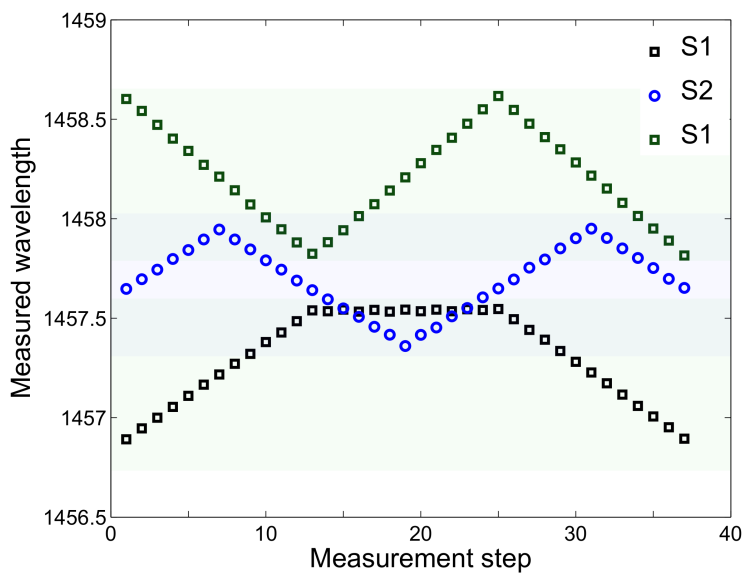

Figure 10. Measured wavelength of an overlapping scenario between three encoded sensors constructed with the orthogonal codes $S 1=[11001000]$ and $S 2=$ [10100001], full overlapping is demonstrated between different sensors while partial overlapping is allowed for sensors with the same encoding.

experiment was performed to validate the detection of 4 sensors sharing the spectrum by pairs. Fig. 11 illustrates the reflection spectrum for the 4 sensors, which were arranged in two branches combining the serial and parallel structures, and the corresponding correlation product from the readout. Fig. 12 shows the trajectories followed for the sensors.

Although the maximum multiplexing capability of a CDMWDM system depends on the number of OOCs used and their particular spectral slot number (N), from Fig. 12 it is possible to see that if in a typical WDM network each sensor requires an operational range of $\pm 2 \mathrm{~nm}$. An equivalent CDM-WDM system, with 2 OOCs, will allow two sensors to operate inside the same spectral region, hence, the number of sensors can be doubled using the proposed encoding technique with very little effect in the wavelength detection accuracy of the system as detailed in section III-B.

\section{B. Experimental Error Discrimination}

The use of encoded FBG sensors allows the identification of two or more sensors under overlapping conditions, which
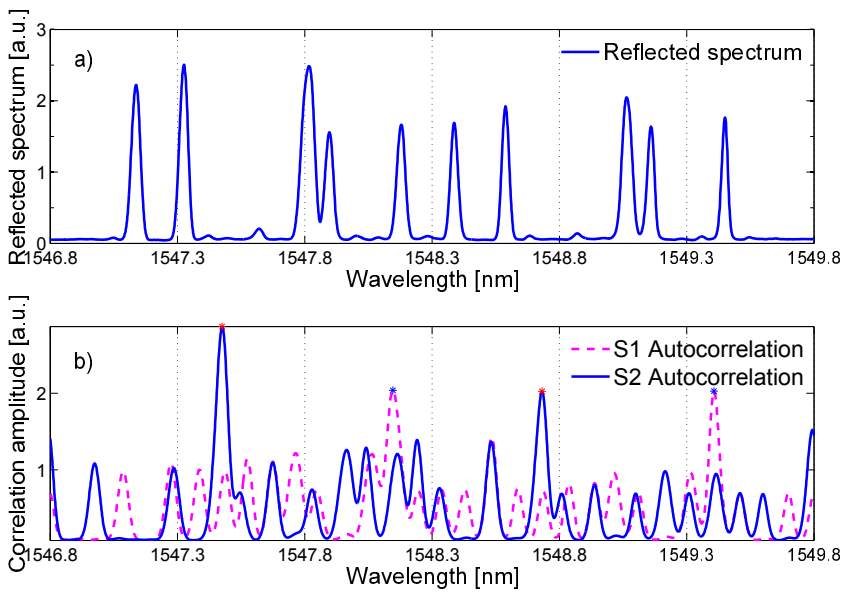

Figure 11. Experimental measurement of the reflection spectrum from four encoded FBG sensors. In a), a readout of the total reflected spectrum under overlapping conditions is measured. In b), their correlation product is computed in order to obtain the ACP corresponding to the central wavelength for each sensor. Maximum values of the ACP depends on the reflectivity of each sensor.

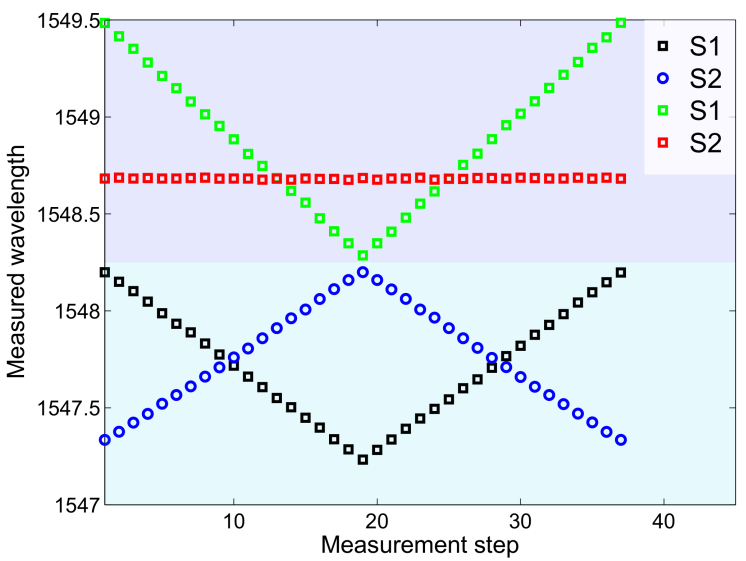

Figure 12. Measured wavelength of an overlapping scenario between four encoded sensors constructed with two orthogonal codes: $S 1=[11001000]$ and $S 2=$ [10100001], full overlapping between encoded FBG sensors and WDM - CDM compatibility are demonstrated.

could increase the number of sensors in a network, but this improvement in the system comes at a cost. There is an additional source of error added to the system due to the effect of other signals in the spectrum influencing the crosscorrelation detection function. As mentioned in section II-B this error is caused by $\mathrm{XC}$ values (corresponding to other signals in the spectrum) added to the ACP value obtained for the proper interrogated sensor.

In order to discriminate this error an experiment with two sensors under overlapping conditions has been performed. The main idea is to compare the wavelength measurements obtained from the overlapping scenario against the ones obtained from individual measurement of the sensors. It is also necessary to isolate the human error from the measurements i.e. the comparison must be carried out between measurements performed at the same spectral position.

Considering the reasons mentioned above, an overlapping scenario have been recreated between two sensors in parallel 

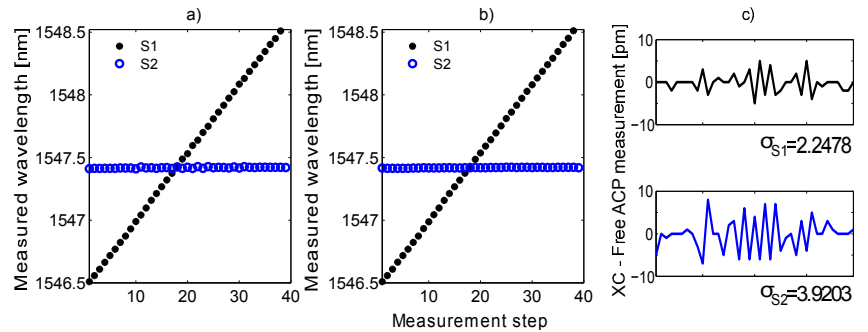

Figure 13. Comparison between the wavelength measurement obtained from the overlapping scenario and the one obtained individually during the same experiment is presented in a) and b) respectively. In c), it is plotted the difference between the two set of measurements.

configuration. In this way, it is possible to switch off one of the sensors thus, reading the reflection spectrum from: sensor $S 1$, sensor $S 2$ and, sensors $S 1$ and $S 2$ simultaneously in the network.

The experiment has been carried out with sensor $S 1$ being swept over $S 2$ and vice-versa. In this way it is possible to obtain the total difference between the individual and the overlapping scenario. The total error in the detection system can be written then as (3):

$$
\sigma E_{T}=\sqrt{\sigma E_{X C}^{2}+\sigma E_{\text {syst }}^{2}}
$$

Where $\sigma E_{X C}$ and $\sigma E_{s y s t}$ are the standard deviation for both, the error due to the cross-correlation, and the systematic error caused by all the components in the system. In Fig. 13 it is depicted the detected wavelength for sensors $S 1$ and $S 2$ when sensor $S 1$ is swept through the spectral position of sensor $S 2$. Fig. 13 a) shows the measurement performed when the two sensors are connected simultaneously to the system. Fig. 13 b) shows the individual measurement through the same experiment. Finally, Fig. 13 c) represents the point by point difference between the two cases obtaining a maximum punctual error of $\pm 7 \mathrm{pm}$. However, the goal is to separate the error caused by the cross-correlation based detection procedure. To do so, we compared the detected wavelength for the stationary sensor $S 2$ with and without the presence of sensor $S 1$ in the spectrum. This is shown in Fig. 14, indeed, Fig. 14 a) delimits clearly the overlapping region between the two sensors (central region) while the external regions denote no overlapping between them. The standard deviation due to the cross-correlation is $\sigma_{X C}=3.8046$. In Fig. 14 b) it is depicted the measured wavelength individually during the same experiment, in this case the standard deviation due to the systematic error is $\sigma_{\text {sys }}=1.3937$. From this, it is computed the error standard deviation due to the cross-correlation of the sensors as 4:

$$
\begin{aligned}
\sigma E_{X C} & =\sqrt{\sigma E_{T}^{2}-\sigma E_{\text {syst }}^{2}} \\
& =\sqrt{3.804^{2}-1.393^{2}}=3.54 \mathrm{pm}
\end{aligned}
$$

\section{CONCLUSION}

In this paper, optical orthogonal codes have been used to design encoded BG sensors. From a set with $n$ OOC we can
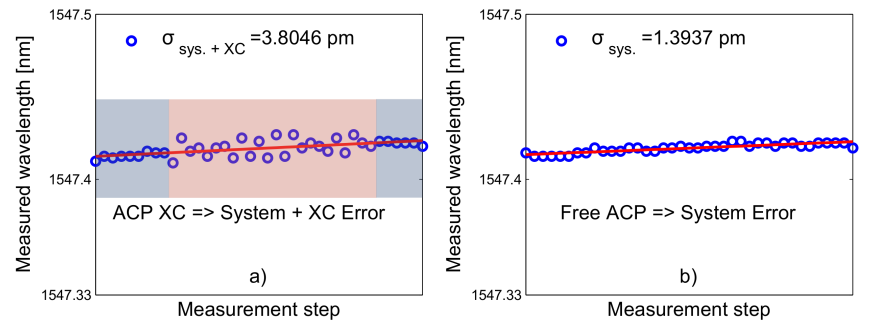

Figure 14. Zoomed wavelength measurement for the fixed sensor in the comparison between an overlapped system (a), and the individual measurement (b). In a), it is possible to discriminate the $\mathrm{XC}$ - from the systematic error when the two sensors start to merge into each other. b) shows only the systematic error for an individual measurement.

obtain $n$ encoded FBG sensors that can be identified even in overlapping scenarios. Due to our design methodology, there is a trade-off relationship between the number of OOC in a set and the spectral length of the FBG devices (the more OOC in the set, the larger the spectral bandwidth of the devices). Nevertheless, with the minimum number of OOC, i.e. 2 , it is possible to double the capacity of the sensing system since two encoded FBG sensors can be allocated in the same spectral region while their central wavelengths are computed and totally distinguishable from each other even under overlapping conditions.

We described theoretically the reflection spectrum for a set of encoded FBG sensors based on their design parameters and the identification function which is based on the correlation product between the codewords. The identification function was implemented and simulation of wavelength detection in overlapping scenarios was performed, error in the detection procedure was found to happen due to the interference between sub-bands when two sensors are overlapping.

A set of 2 encoded sensors was manufactured as SI-FBGs, ensuring that the sub-bands belonging to a sensor are affected in the same way by the environmental changes surrounding the optical fiber. The manufacturing method was performed in a point by point writing exposure of the fiber's core to an UV laser through a phase mask. It was obtained a very good agreement between designed and manufactured devices. Experimental validation of the proposed sensors under overlapping scenarios was carried out using serial and parallel configuration. Devices connected in serial configuration should have low reflectivity, $(<60 \%)$, due to shadowing effect between sensors working at the same wavelength while the only consideration when working in parallel configuration is related to the losses of the passive splitter used to connect the sensors. It was experimentally verified the wavelength discrimination in both cases and additional experiments were performed to demonstrate the compatibility between CDM and WDM multiplexing techniques. The encoded FBG sensors proved to be totally recognizable not only from other sensors but also with shifted versions of itself.

We have shown the existence of an error in the wavelength detection in the system due to the interference between XC and ACP signals from the same spectrum. To address this, experimental measurements were performed in order to compare the detected wavelength values in an overlapping scenario 
against individual measurements performed during the same experiment. A total error in the system was found to have a standard deviation of $3.804 \mathrm{pm}$, being the component due to the cross-correlation between the sensors of $3.54 \mathrm{pm}$. This is a low value for the total error in the system meaning $\pm 3 \mu \epsilon$ for strain measurement and $\pm 0.3^{\circ} \mathrm{C}$ for temperature measurements.

\section{REFERENCES}

[1] A.Kersey et al., "Fiber grating sensors," Lightwave Technology, Journal of, vol. 15, no. 8, pp. 1442-1463, Aug 1997.

[2] T.Erdogan, "Fiber grating spectra," Lightwave Technology, Journal of, vol. 15, no. 8, pp. 1277-1294, Aug 1997.

[3] M.Tateda and T.Horiguchi, "Advances in optical time domain reflectometry," Lightwave Technology, Journal of, vol. 7, no. 8, pp. 1217-1224, Aug 1989.

[4] K.Yuksel et al., "Optical frequency domain reflectometry: A review," in Transparent Optical Networks, 2009. ICTON 09. 11th International Conference on, June 2009, pp. 1-5.

[5] D.Liu et al., "A Fiber Bragg Grating Sensor Network Using an Improved Differential Evolution Algorithm," Photonics Technology Letters, IEEE, vol. 23, no. 19, pp. 1385-1387, Oct 2011.

[6] H.Jiang et al., "Wavelength Detection in Spectrally Overlapped FBG Sensor Network Using Extreme Learning Machine," Photonics Technology Letters, IEEE, vol. 26, no. 20, pp. 2031-2034, Oct 2014.

[7] J.Chen et al., "Wavelength detection in FBG sensor networks using least squares support vector regression," Journal of Optics, vol. 16, no. 4, 2014. [Online]. Available: http://stacks.iop.org/2040$8986 / 16 / \mathrm{i}=4 / \mathrm{a}=045402$

[8] A.Kersey et al., "Low-crosstalk code-division multiplexed interferometric array," Electronics Letters, vol. 28, no. 4, pp. 351-352, Feb 1992.

[9] K.Koo et al., "Dense wavelength division multiplexing of fibre Bragg grating sensors using CDMA," Electronics Letters, vol. 35, no. 2, pp. 165-167, Jan 1999.

[10] J.Ko et al., "Fiber Bragg grating sensor network based on code division multiple access using a reflective semiconductor optical amplifier," Microwave and Optical Technology Letters, vol. 52, no. 2, pp. 378-381, 2010. [Online]. Available: http://dx.doi.org/10.1002/mop.24913

[11] H.Lee, "Multiple fiber Bragg grating sensor system using code-division multiple access," Appl. Opt., vol. 41, no. 25, pp. 5245-5248, Sep 2002. [Online]. Available: http://ao.osa.org/abstract.cfm?URI=ao-41-25-5245

[12] C.Triana et al., "Optical code division multiplexed fiber Bragg grating sensing networks," vol. 9634, 2015, pp. 963 478-963 478-4. [Online]. Available: http://dx.doi.org/10.1117/12.2194995

[13] F.Chung et al., "Optical orthogonal codes: design, analysis and applications," Information Theory, IEEE Transactions on, vol. 35, no. 3, pp. 595-604, May 1989.

[14] C.Huang et al., "Demodulation of Fiber Bragg Grating Sensor Using Cross-Correlation Algorithm," Photonics Technology Letters, IEEE, vol. 19, no. 9, pp. 707-709, May 2007.

[15] S.Ayotte et al., "Experimental verification and capacity prediction of FEOCDMA using superimposed FBG," Lightwave Technology, Journal of, vol. 23, no. 2, pp. 724-731, Feb 2005.

[16] R.Baños et al., "Rectangular Global Envelope Super Structured FBGs for Multiband Coherent OCDMA," Photonics Technology Letters, IEEE, vol. 25, no. 5, pp. 512-514, March 2013.

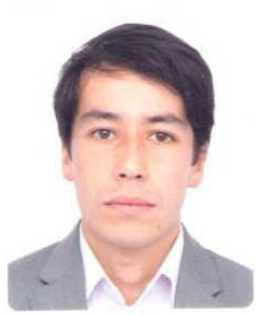

Andrés Triana was born in Bogotá, Colombia, in 1988. He received the Bachelor's degree in electronics engineering and the M.Eng. degree in industrial automation, both from the Universidad Nacional de Colombia, Bogotá, in 2011 and 2013, respectively. He is currently working towards the Ph.D. degree in communications engineering at the Optical and Quantum Communication Group (OQCG) from Universitat Politècnica de València and Universidad Nacional de Colombia. In 2014, he joined the OQCG as a Researcher and he has been involved in the FBGs sensors field. His research interests include the design and fabrication of FBGs, optical sensors and microwave photonics applications.

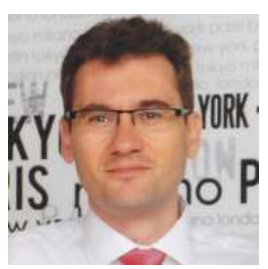

Daniel Pastor was born in Elda, Spain, on November 5, 1969. He received the Ingeniero de Telecomunicación degree and the Doctor Ingeniero de Telecomunicación (Ph.D.) degree from the Universitat Politècnica de València (UPV), Valencia, Spain, in 1993 and 1996, respectively. He joined the Departamento de Comunicaciones, UPV, in 1993, where he was with the Optical and Quantum Communications Group. He became Associate Professor in 1999, and Full Professor in 2010, carrying out his teaching activities at the Telecommunications Engineering Faculty. He is coauthor of more than 190 papers in journals and international conferences in the fields of optical delay line filters, Fiber Bragg Gratings, Microwave Photonics, Wavelength-Division-Multiplexing (WDM) and Optical Code Division Multiple Access (OCDMA) devices and techniques. His current technical interests include complex Fiber Bragg Grating (FBG) design for signal processing, fiber sensors and OCDMA, and Photonic Integrated Circuits (PICs) applied to telecommunication and sensing.

Margarita Varón was born in Cartagena, Colombia. She received the B.Eng. degree in electronic engineering from Universidad Javeriana, Bogotá, Colombia, in 2000, the M.Sc. degree in optical communications and photonic technologies from Politecnico di Torino, Turin, Italy, in 2003, and the Ph.D. degree in optoelectronics from ISAE-SUPAERO, Toulouse, France, in 2008. Since 2010, she has been with Universidad Nacional de Colombia, in Bogotá Colombia, where she is currently an Associate Professor. Her main areas of research interest are optoelectronics, particularly optical fiber sensors, optoelectronic oscillators, microwave photonics and optical communications. 